\title{
LOW TEMPERATURE PHASES OF MICROCRYSTALLINE $\mathrm{FeCl}_{3}$
}

\author{
F. J. LITTERST, W. BRÖLL and G. M. KALVIUS \\ Physik Department der Technischen Universität München \\ D-8046 Garching, James-Franck-Str., Germany
}

\begin{abstract}
Résumé. - Les spectres Mössbauer de $\mathrm{FeCl}_{3}$ déposé à basse température à partir de la vapeur montrent deux doublets quadrupolaires au-dessus de $6,5 \mathrm{~K}$ et des éclatements magnétiques hyperfins en dessous de $6,5 \mathrm{~K}$. Par chauffage à $300 \mathrm{~K}$, les échantillons peuvent être transformés irréversiblement en l'état cristallin normal. En dessous de leurs températures de transition magnétique, les spectres des phases trempées à partir de la vapeur ainsi que ceux de la phase cristalline normale montrent des effets de relaxation qui sont attribués à des fluctuations superparamagnétiques. Par opposition aux halogénures ferreux amorphes, les courbes d'aimantation des phases de $\mathrm{FeCl}_{3}$ trempées à partir de la vapeur ont la même dépendance fonctionnelle que la phase cristalline. On

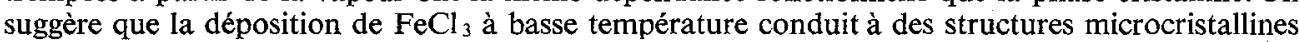
plutôt qu'amorphes.
\end{abstract}

\begin{abstract}
Mössbauer spectra of $\mathrm{FeCl}_{3}$, vapour deposited at low temperatures, show two quadrupole doublets above $6.5 \mathrm{~K}$ and magnetic hyperfine splittings below $6.5 \mathrm{~K}$. Upon heating to $300 \mathrm{~K}$ the samples can be transformed irreversibly to the normal crystalline phase. Below their magnetic transition temperatures the spectra of both vapour quenched phases and of the normal crystalline phase exhibit relaxation effects which are interpreted as superparamagnetic fluctuations. In contrast to the amorphous ferrous halides, the magnetization curves of the vapour quenched phases of $\mathrm{FeCl}_{3}$ show the same functional dependence as the crystalline phase. It is suggested that the low temperature deposition of $\mathrm{FeCl}_{3}$ leads to microcrystalline rather than to amorphous structures.
\end{abstract}

1. Introduction. - It has been shown that the condensation of ferrous halides from a molecular beam onto a helium-cooled substrate leads to the formation of a non-crystalline phase [1]. The magnetic properties of these samples as studied with the Mössbauer effect [2], are markedly different from those of the crystalline compounds and have been discussed within the framework of published theories of amorphous magnetism $[3,4,5]$. The present paper discusses Mössbauer spectroscopic investigations of $\mathrm{FeCl}_{3}$ samples prepared in a similar manner from a molecular beam. It will be shown that the behaviour of these $\mathrm{FeCl}_{3}$ samples is very different from those of the ferrous halides.

2. Experimental. - Samples of anhydrous ${ }^{57} \mathrm{FeCl}_{3}$ were condensated from a molecular beam onto an Al-foil kept at temperatures $<10 \mathrm{~K}$ in a vacuum of $<5 \times 10^{-8}$ torr. The deposition process was continuously monitored by the transmission of the $6 \mathrm{keV}$ $\mathrm{X}$-rays from a $30 \mathrm{mCi}{ }^{57} \mathrm{Co}(\mathrm{Cu})$ source through the sample. Different samples were thus prepared with thicknesses between $\sim 750 \AA$ and $30000 \AA$ and served as the absorber in a Mössbauer transmission experiment. The sublimation temperature in the molecular

(*) Work supported by the «Bundesministerium für Forschung und Technologie $\gg$. beam oven was usually kept at $360 \mathrm{~K}$. It is known [6] from electron diffraction measurements that at this temperature the vapour consists totally of dimer molecules $\mathrm{Fe}_{2} \mathrm{Cl}_{6}$. Higher temperatures which should lead to the sublimation of monomer $\mathrm{FeCl}_{3}$ could not be applied because of the onset of decomposition into $\mathrm{FeCl}_{2}$ and $\mathrm{Cl}_{2}$. Mössbauer spectra were taken between 2.6-300 $\mathrm{K}$ without removing the samples from the cryostat. Details of the apparatus will be described elsewhere [7].

3. Results. - At temperatures $\gtrsim 6.5 \mathrm{~K}$ spectra consisting of four partially resolved resonance lines were observed. They are interpreted as the quadrupole pattern of two non-identical Fe sites, labeled A and B in figure 1. The splittings are $\Delta E_{\mathrm{Q}}(\mathrm{A})=1.74 \mathrm{~mm} / \mathrm{s}$ and $\Delta E_{\mathrm{Q}}(\mathrm{B})=0.86 \mathrm{~mm} / \mathrm{s}$, the isomer shifts are IS(A) $=0.37 \mathrm{~mm} / \mathrm{s}$ and IS(B) $=0.47 \mathrm{~mm} / \mathrm{s}$ at $20 \mathrm{~K}$ against an absorber of $\mathrm{Fe}$ metal at $300 \mathrm{~K}$. These values are in marked contrast to the well-known hyperfine (hf) parameters of crystalline $\mathrm{FeCl}_{3}$ which, above its Néel temperature $T_{\mathrm{N}}=8.9 \mathrm{~K}$, shows a slightly broadened, single line $\left(\Delta E_{\mathrm{Q}} \lesssim 0.05 \mathrm{~mm} / \mathrm{s}\right)$ with IS $=0.57 \mathrm{~mm} / \mathrm{s}$. The relative intensities of the two quadrupole spectra (area under the lines) is dependent on conditions during sample preparation. Sublimation with higher deposition rates than $\sim 50 \AA / \mathrm{min}$ favours formation of the doublet $B$ which has the smaller 


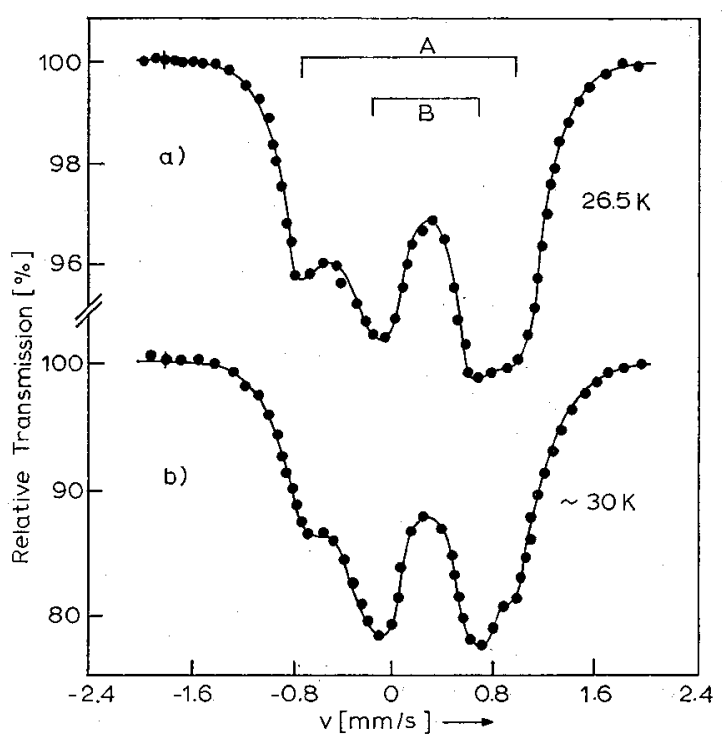

FIG. 1. - Spectra of two samples of $\mathrm{FeCl}_{3}$, deposited from a molecular beam onto $a<10 \mathrm{~K} \mathrm{Al}$ foil for $T>T_{\mathrm{m}}$. Sample a) (thickness $\sim 5000 \AA$ ) was deposited at a rate of $25 \AA / \mathrm{min}$, sample $b$ ) (thickness $30000 \AA$ ) at a rate of $300 \AA / \mathrm{min}$.

quadrupole splitting. Below about $6.5 \mathrm{~K}$ a magnetic hf pattern with rather broad resonance lines is observed (Fig. 2). The temperature ( $\left.T_{\mathrm{m}}\right)$ at which the magnetic pattern begins to appear is again dependent on the preparation condition and sample thickness. Values range from $4 \mathrm{~K}$ (for a sample with $\sim 750 \AA$ thickness and a deposition rate of $\sim 10 \AA / \mathrm{min}$ ) to $6.5 \mathrm{~K}$ (for samples thicker than $\sim 13000 \AA$ and deposition rates of $60-300 \AA / \mathrm{min}$ ). In general, magnetic spectra seem to appear for the vapour quenched samples only well

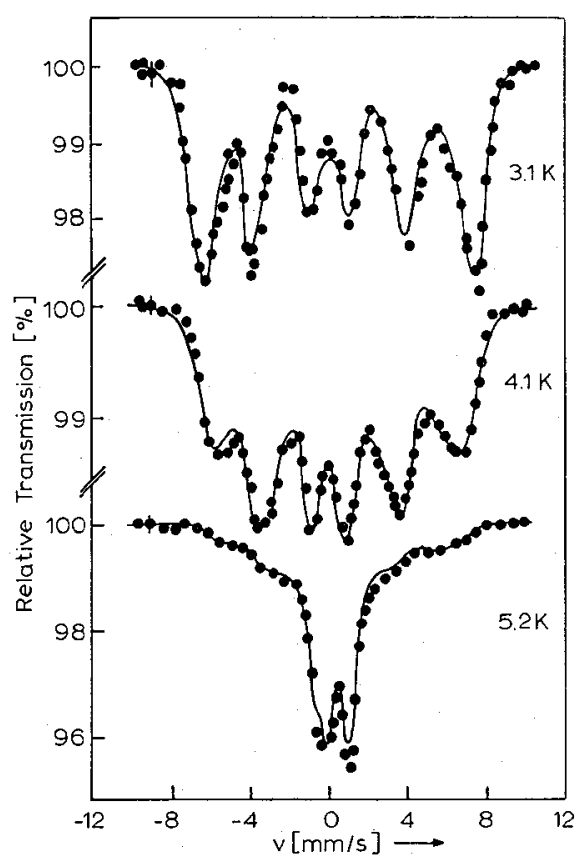

FIG. 2. - Spectra of $\mathrm{FeCl}_{3}$ (absorber thickness $13000 \AA$ ) deposited from a molecular beam onto $a<10 \mathrm{~K} \mathrm{Al}$ foil at a rate of $80 \AA / m i n$ for $T<T_{\mathrm{m}}$. below the Néel temperature of crystalline $\mathrm{FeCl}_{3}$. The magnetic spectra can be interpreted as two 6-line patterns with identical magnetic hf fields and the quadrupole splittings and isomer shifts found at higher temperatures for the sites $\mathrm{A}$ and $\mathrm{B}$. However, a rather substantial line broadening (FWHM $\gtrsim 0.5 \mathrm{~mm} / \mathrm{s}$ ) has to be assumed for such a fit. This broadening, together with the strong enhancement of the $m_{\mathrm{e}}=\frac{1}{2} \rightarrow m_{\mathrm{g}}=-\frac{1}{2}$ and $m_{\mathrm{e}}=-\frac{1}{2} \rightarrow m_{\mathrm{g}}=\frac{1}{2}$ hf transitions is taken as evidence for spin fluctuations with correlation frequencies comparable to the nuclear Larmor frequency. For least squares curve fitting procedures a relaxation model given by van der Woude and Dekker $[8,9]$ was used. This model is considered to be especially appropriate in the vicinity of a magnetic transition point where the Weiss molecular field theory is a good approximation. The electronic spins are thought to flip stochastically between discrete Zeeman levels due to the interaction with fluctuating local fields. The relaxation frequencies calculated from the curves fitted to the observed hf spectra are strongly temperature dependent. They are best interpreted in terms of a fluctuation of the magnetization of superparamagnetic particles. Such fluctuations of magnetization in particles with volume $V$ and anisotropy energy density $K$ occur according to Jacobs and Bean [10] with a characteristic flipping time given by

$$
\tau_{\mathrm{F}}=\tau_{0} \cdot \exp (K \cdot V / k T) .
$$

The time $\tau_{0}$ can be understood as the reciprocal of the Larmor frequency of the magnetization $M$ in an effective molecular field $H_{\mathfrak{n}} \propto K V / M$. If $\tau_{\mathrm{F}} \ll \tau_{\mathrm{L}}$, the Larmor precession period of the nuclear magnetic moment in the effective hyperfine field, the nuclear Zeeman splitting will disappear and a non-magnetic spectrum will be observed. This will occur if the energy barrier $K . V$ becomes small enough which occurs usually for particles below $100 \AA$ diameter. The value of $\tau_{0}$ may be estimated to be on the order of $10^{-10}-10^{-12} \mathrm{~s}$. Its exact magnitude is not a stringent condition for the occurrence of superparamagnetic behaviour, since the exponential term dominates $\tau_{\mathrm{F}}$. Superparamagnetism has been studied by Mössbauer spectroscopy in considerable detail for fine particles of different iron oxides by various authors $[11,12,13]$. It could be shown that the above relaxation theories lead to a good understanding of the Mössbauer spectra. For our samples we find a nearly constant value of $K . V=6 \times 10^{-15}$ erg for absorbers thicker than $13000 \AA$ in the temperature regime $T \lesssim 0.9 T_{\mathrm{m}}$ under the assumption of $\tau_{0} \simeq 10^{-11} \mathrm{~s}$. Near $T_{\mathrm{m}}$ the observed decrease of $K . V$ reflects the expected lowering of anisotropy constant. Thinner absorbers give in general a somewhat lower value of $K . V$. Figure $3 a$ gives the temperature dependence of the mean hf field as returned from the relaxation fits. It should be noticed that the saturation value $(T \rightarrow 0)$ of the hf field is only $420 \mathrm{kOe}$ and thus much smaller than the value of $495 \mathrm{kOe}$ reported [14] for crystalline $\mathrm{FeCl}_{3}$. 

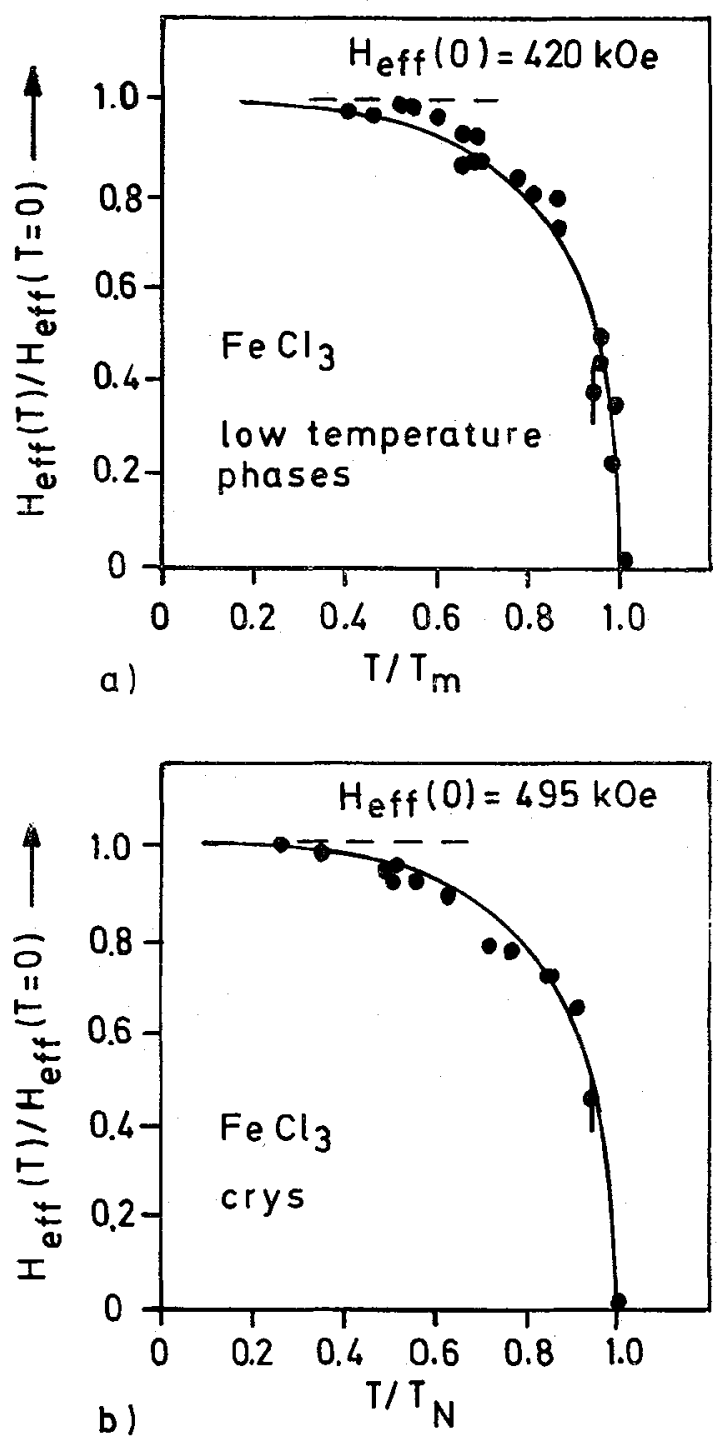

FIG. 3. - Temperature dependence of hf magnetic field for $\mathrm{FeCl}_{3}$ deposited from a molecular beam onto $a<10 \mathrm{~K} \mathrm{Al}$ foil a) and for $\mathrm{FeCl}_{3}$ converted into the normal crystalline phase $b$ ).

Upon heating to about $100 \mathrm{~K}$, phase A gradually converts irreversibly into phase B. At the same temperature a partial formation of the normal crystalline phase is also observed (Fig. 4). Complete crystallization can be achieved only for absorbers thicker than $\sim 10000 \AA$ while thinner samples show only partial crystallization even after annealing at $330 \mathrm{~K}$ for several days. The resonance spectra as a function of temperature for such recrystallized absorbers is plotted in figure 5. They show the expected single resonance line (unresolved quadrupole splitting) above $T_{\mathrm{N}}$. The value of the Néel temperature $T_{\mathrm{N}}=8.90 \pm 0.05 \mathrm{~K}$ was determined by thermal scanning [15] and is in excellent agreement with the results of Stampfel et al. [14] for single crystals of $\mathrm{FeCl}_{3}$. In the course of this work we have also investigated the Mössbauer spectra of the polycrystalline $\mathrm{FeCl}_{3}$ which was used in generating the molecular beam. The spectra of the polycrystalline

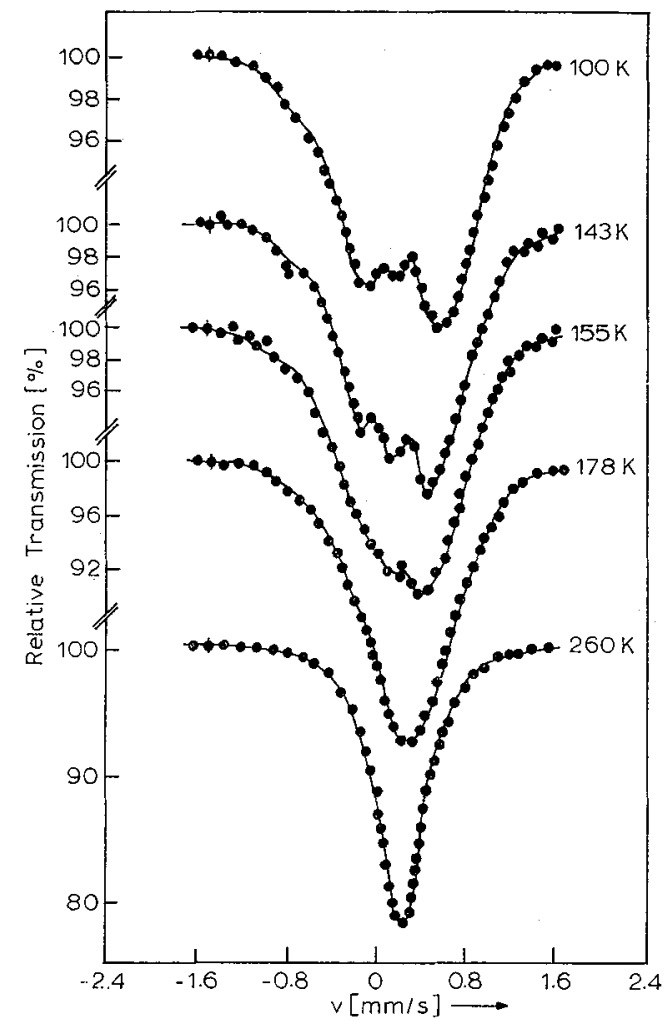

Fig. 4. - Spectra of $\mathrm{FeCl}_{3}$ (absorber thickness $30000 \AA$ ) deposited from a molecular beam onto $a<10 \mathrm{~K} \mathrm{Al}$ foil at a rate of $300 \AA / \mathrm{min}$, for various temperatures above $100 \mathrm{~K}$.

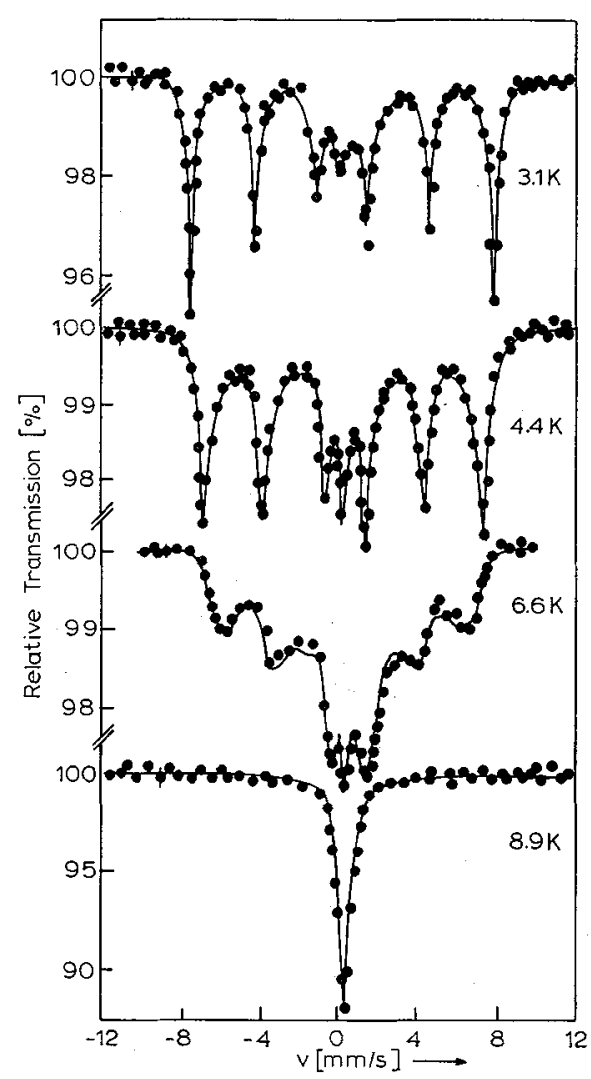

FTG. 5. - Low temperature spectra of vapour deposited $\mathrm{FeCl}_{3}$ (see Fig. 2) after annealing at $330 \mathrm{~K}$. 
material are similar to those shown in figure 5 and exhibit also relaxational broadening below $T_{\mathrm{N}}$. Especially thin absorbers show a pronounced paramagnetic single line in the middle of the spectrum. Those spectra could be fitted with the same superparamagnetic relaxation procedure described above. A Gaussian distribution of particle sizes $0.5 \leqslant V / \bar{V} \leqslant 1.5$ around the mean volume $\bar{V}$ was required to obtain a good fit. The temperature dependence of the hf field obtained from this fit is given in figure $3 b$. It shows the same functional behaviour as that of the vapour quenched sample, but gives the expected higher saturation value of the hf field. The energy barriers $K . V$ for the flipping of the particle magnetization are $6.5-7.5 \times 10^{-15}$ erg for samples thicker than $\sim 10000 \AA$ in the temperature range up to $\sim 0.9 T_{\mathrm{N}}$.

4. Discussion. - The well-known crystalline phase of $\mathrm{FeCl}_{3}$ forms a rhombohedral bimolecular lattice where $\mathrm{Fe}^{3+}$ ions possess a nearly octahedral surrounding [16]. The quadrupole splitting is thus very small and cannot be resolved. The isomer shifts of the low temperature vapour quenched phases $\mathbf{A}$ and $\mathbf{B}$ are lower than that found for the crystalline phase indicating a higher contact electron density. This may arise from a lower $\mathrm{Cl}^{-}$coordination of the $\mathrm{Fe}^{3+}$ ion when compared to that of the octahedral surrounding in the crystalline $\mathrm{FeCl}_{3}$. From geometrical reasons a tetrahedral coordination appears favourable for a ratio of the ionic radii $r_{\mathrm{Fe}^{3}} / r_{\mathrm{Cl}^{-}} \simeq 0.35$ [17]. Since $\mathrm{FeCl}_{3}$ vapour below $1000 \mathrm{~K}$ is formed from $\mathrm{Fe}_{2} \mathrm{Cl}_{6}$. molecules [6] (see Fig. 6), we assume that the tetrahedral coordination of the $\mathrm{Cl}^{-}$ions around $\mathrm{Fe}^{3+}$ as present

\section{$\mathrm{Fe}_{2} \mathrm{Cl}_{6}$}
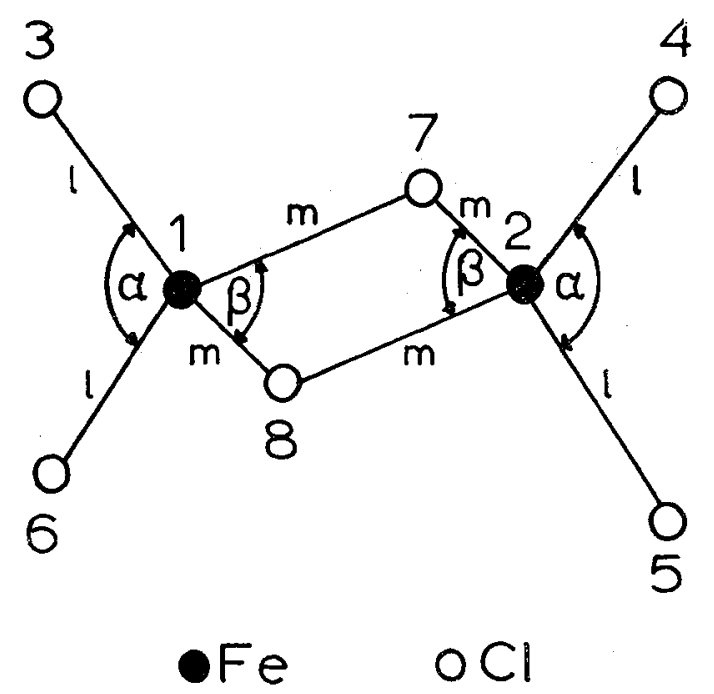

FIG. 6. $-\mathrm{Fe}_{2} \mathrm{Cl}_{6}$ molecule (see ref. [6]). Bond lengths: $l=2.11 \pm 0.03 \AA ; \quad m=2.28 \pm 0.03 \AA$. Bond angles : $\alpha=(128 \pm 3)^{0}, \beta=(92 \pm 3)^{0}$. Ions $1,2,3,4,5,6$ form a plane perpendicular to $1,2,7,8$. in this dimer molecule is frozen during the rapid condensation. Direct formation of the known crystalline phase from the dimer molecules could not be observed. The electric field gradient at the $\mathrm{Fe}^{3+}$ site of the dimer molecule can be estimated using a simple point charge model [18] with the parameters given in figure 6. Higher than nearest neighbour influences were neglected. A splitting $\Delta E_{\mathrm{Q}} \simeq 1.6 \pm 0.2 \mathrm{~mm} / \mathrm{s}$ was calculated. This value is in agreement with that found for phase $\mathrm{A}: \Delta E_{\mathrm{Q}}(\mathrm{A})=1.74 \mathrm{~mm} / \mathrm{s}$. The splitting of the doublet $\mathrm{B}$ with $\Delta E_{\mathrm{Q}}(\mathrm{B})=0.86 \mathrm{~mm} / \mathrm{s}$ is obviously caused by a slightly higher symmetrical coordination around the $\mathrm{Fe}^{3+}$ This could come about if the angle $\alpha$ in figure 6 were reduced. Such a deformation is expected if long chains are formed by $\mathrm{Fe}_{2} \mathrm{Cl}_{6}$ molecules linked together end by end. The observed gradual transformation to phase B upon annealing may be regarded as an indication for the formation of such chains. The reduced value for the saturation hf field for both condensed molecular phases when compared to crystalline $\mathrm{FeCl}_{3}$ - which itself is already rather small with respect to most ionic ferric ions - is in agreement with the increased population of the $4 \mathrm{~s}$ shell deduced above from the increase in contact density (decrease of IS). It had been mentioned earlier, that Mössbauer effect studies of the magnetic properties of the dihalides $\mathrm{FeF}_{2}, \mathrm{FeCl}_{2}$ and $\mathrm{FeBr}_{2}$ proved the existence of noncrystalline phases if these materials were prepared with the same method used here. These ferrous halides [2] and other non-crystalline magnets [15] exhibit a distribution of hf fields of $10-20 \%$ around its mean value. In contrast the magnetically split spectra of the condensed phases of $\mathrm{FeCl}_{3}$ set an upper limit of $\sim 5 \%$ for the distribution of the hf field around its mean value. The comparison of the normalized magnetization curve (Fig. $3 a$ ) of the condensed phases of $\mathrm{FeCl}_{3}$ with those of the non-crystalline ferrous halides [2] again show a significant difference : Due to fluctuations in the local exchange integrals $[3,4]$ resp. in the anisotropy fields [5] a considerably less steep rise of the hf field below the magnetic ordering temperature is characteristic for amorphous magnets. The mean magnetization curve of the low temperature vapour quenched phases of $\mathrm{FeCl}_{3}$, however, still has the same functional dependence as that of crystalline $\mathrm{FeCl}_{3}$ (Fig. 3b). From the apparent absence of fluctuations in exchange integrals we conclude that the low temperature phases cannot be interpreted as being amorphous in the sense of a random network amorphicity [20] which is the basis of the magnetic theories of references [3-5]. It is our opinion that we have here an example of a cluster type or microcrystalline material with particles on the order of 20-100 $\AA$ diameter. Such a microcrystalline structure is sometimes also called non-crystalline [20]. Evidence for microcrystalline structural units comes from the observed superparamagnetic relaxation. Although the calculated values of the flipping barriers $K . V$ should be taken with care because of the very simple model applied, 
they can be used for a rough estimate of the average particle size. Taking $K=5 \times 10^{4}-1 \times 10^{5} \mathrm{erg} / \mathrm{cm}^{3}$ which is the appropriate range of values [21] for small particles of $\mathrm{FeF}_{3}$ we find particle diameters of the order of $40-60 \AA$.

The apparent decrease of $T_{\mathrm{m}}$ with sample thickness is connected with a decrease of flipping barriers and thus represents the expected decrease in blocking temperature of the superparamagnetic fluctuations.

The samples transformed to the normal crystalline state still exhibit superparamagnetic behaviour. Probably large crystals cannot be formed upon annealing due to the small dimensions of our samples. This is reflected also by the apparent reluctance of thin absorbers to transform to the normal crystalline state at all. Furthermore, it appears that even the usual chemical procedure for the preparation of $\mathrm{FeCl}_{3}$ leads to rather small crystallites. Superparamagnetism in crystalline $\mathrm{FeCl}_{3}$ can only be avoided if care is taken to grow large crystals.

Acknowledgment. - We wish to thank Prof. A. J. F. Boyle (University of Western Australia) for many helpful discussions.

\section{References}

[1] Boyle, A. J. F., Kalvius, G. M., Gruen, D. M., ClifTON, J. R., MCBETH, R. L., J. Physique Colloq. 32 (1971) C 1-224.

[2] Litterst, F. J., Kalvius, G. M., Boyle, A. J. F., Proc. 19th Conf. Magnetism and Magnetic Materials (1973) Boston, AIP Conf. Proc. 18 part 1, 616, Graham C. D. Jr., Rhyne J. J., eds.

[3] Simpson, A. W., Brambley, D. R., Phys. Stat. Sol. (b) 49 (1972) 685, and references given there.

[4] Handrich, K., Phys. Stat. Sol. 32 (1969) K 55.

[5] Harris, R., Pitschke, M., Zuckermann, M. J., Phys. Rev. Lett. 31 (1973) 160.

[6] Zasorin, E. Z., Rambidi, N. G., Akishin, P. A., J. Struct. Chem. 4 (1963) 836.

[7] BröLL, W., LitTERST, F. J., to be published.

[8] Van der Woude, F., DekKer, A. J., Phys. Stat. Sol. 9 (1965) 775.

[9] Van der Woude, F., Dekker, A. J., Phys. Stat. Sol. 13 (1966) 181.

[10] Jacobs, I. S., Bean, C. P., in Magnetism III (1963) 271, Acad. Press, London, New York, Rado, Suhl, eds.

[11] Kündig, W., Bömmel, H., Constabaris, G., LindQuist, R. H., Phys. Rev. 142 (1966) 327.
[12] MCNab, T. K., Fox, R. A., Boyle, A. J. F., J. Appl. Phys. 39 (1968) 5703.

[13] Topsøe, H., Dumesic, J. A., Boudart, M., J. Physique Colloq. 35 (1974) C 6.

[14] Stampfel, J. P., Oosterhuis, W. T., Window, B., de S. Barros, F., Phys. Rev. B 8 (1973) 4371.

[15] Dunlap, B. D., Dash, J. G., Phys. Rev. 155 (1967) 460.

[16] Wykoff, R. W. G., Crystal Structures 2 (1965) 45 (Wiley, N.Y.).

[17] ZarzyckI, J., in Physics of Non-Crystalline Solids, Proc. Int. Conf. Delft (1964) 525 (North Holland, Amsterdam) Prins J. A., ed.

[18] Danon, J., in Chemical Applications of Mössbauer Spectroscopy (1968) 177 (Acad. Press New York, London), Goldanskii V. I., Herber R. H., eds.

[19] Sharon, T. E., Tsuei, C. C., Solid State Commun. 9 (1971) 1923.

[20] Stevels, J. M., in Physics of Non-Crystalline Solids, Proc. Int. Conf. Delft (1964) 497 (North Holland, Amsterdam) Prins J. A., ed.

[21] Levinson, L. M., Luban, M., Shtrikman, S., Phys. Rev. 177 (1966) 864. 